\title{
Non-Invasive Technique-Based Novel Corona(COVID-19) Virus Detection Using CNN
}

\author{
N. R. $\operatorname{Raajan}^{1}$ (1) V. S. Ramya Lakshmi ${ }^{1}$ Natarajan Prabaharan $^{1}$
}

Received: 14 May 2020/Revised: 18 June 2020/ Accepted: 17 July 2020/Published online: 30 July 2020

(C) The National Academy of Sciences, India 2020

\begin{abstract}
A novel human coronavirus 2 (SARS-CoV-2) is an extremely acute respiratory syndrome which was reported in Wuhan, China in the later half 2019. Most of its primary epidemiological aspects are not appropriately known, which has a direct effect on monitoring, practices and controls. The main objective of this work is to propose a high speed, accurate and highly sensitive CT scan approach for diagnosis of COVID19. The CT scan images display several small patches of shadows and interstitial shifts, particularly in the lung periphery. The proposed method utilizes the ResNet architecture Convolution Neural Network for training the images provided by the CT scan to diagnose the coronavirus-affected patients effectively. By comparing the testing images with the training images, the affected patient is identified accurately. The accuracy and specificity are obtained $95.09 \%$ and $81.89 \%$, respectively, on the sample dataset based on CT images without the inclusion of another set of data such as geographical location, population density, etc. Also, the sensitivity is obtained $100 \%$ in this method. Based on the results, it is evident that the COVID-19 positive patients can be classified perfectly by using the proposed method.
\end{abstract}

Keywords Coronavirus - CT scan · Diagnosis ·

Convolution neural network

Mathematics Subject Classification 94B10 - 62M45

N. R. Raajan

nrraajan@gmail.com

V. S. Ramya Lakshmi

ramyasrinivasan18@gmail.com

1 Present Address: School of EEE, SASTRA Deemed University, Thanjavur, Tamil nadu, India

\section{Significant Statement}

1. This work proposes an expedite, accurate imagining approach to diagnose COVID19.

2. Small shadow patches, interstitial shifts in the lungs due to the virus are traced through $\mathrm{CT}$ images and are trained using ResNet architecture in CNN to obtain 95.09\% accuracy.

3. When compared with RT-PCR method, this gives high accuracy, sensitivity, specificity.

COVID19 is an acute, often critical respiratory disorder induced by a novel SARS-CoV2 coronavirus. COVID-19 is confirmed by the Reverse Transcription Polymerase Chain Reactors (RT-PCR) or breathing gene sequences or blood samples as the primary predictor of hospitalization [1]. It can be seen that many researchers are undergoing treatment and vaccination research for coronavirus [2-4]. As radiology is frequently the first destination for patients with an immediate febrile condition, the treatment of such patients plays a significant role in the detection and infection control of the particular patients [5]. The deep learning was applied to the detection of cancer cells [6-13] and tumour [14] from CT scan images are performed. This work supports a novel computer-aided diagnosis system for Covid-19 detection based on a Deep Convolution Neural Network (D-CNN). Here, ResNet-16 network architecture is used for training and labelling the dataset. In the early stage of COVID-19, images indicate many irregular patches and cross-sectional shifts in the lungs. As the disease progresses, multiple ground glass shadows and cross-sectional shift gets developed in the images. Lung consolidation may happen in severe cases. Pleural effusion is rarely observed in COVID-19 patients. 
The sequential procedure for automatic diagnosis of COVID19 by CNN is given in Fig. 1. The fine-tuned Convolution Neural Network is implemented for automatic diagnose of COVID-19 patient. In this method, the two types of dataset are considered. One is the COVID-19 image dataset (Fig. 2a) has taken from the GitHub repo [15]. The sample dataset of the healthy patients is taken from the Kaggles CT dataset (Fig. 2b) as the reference images or training dataset. Training the $\mathrm{CNN}$ is performed by automated segmentation of the lung image to extract the patches of corona, non-corona and background images. Initially, the CT scan image is divided into blocks, and the average and variance are calculated for each block from Eqs. 1 and 2. With these calculations, the pixels of whole image are classified.

$$
\begin{aligned}
& A V G_{x, y}=\frac{1}{k^{2}} \sum_{m=0}^{k-1} \sum_{n=0}^{k-1} L(x+m, y+n) \\
& V A R_{x, y}=\frac{1}{k^{2}} \sum_{m=0}^{k-1}\left(\sum_{n=0}^{k-1} L(x+m, y+n)-A V G_{x, y}\right) \\
& A=\frac{1}{W \times H} \sum_{x=0}^{W} \sum_{y=0}^{H} A V G_{x, y} \\
& V=\frac{1}{W \times H} \sum_{x=0}^{W} \sum_{y=0}^{H} V A R_{x, y}
\end{aligned}
$$

$\mathrm{L}$ is the lungs image with the size $\mathrm{x} \times \mathrm{y} ; A V G_{x, y}$ is the average of a block with a size of $\mathrm{k} \times \mathrm{k}$. Corresponding to entire image, Eqs. (1) and (2) are substituted in Eqs. (3) and (4). When the $A v g_{x y}$ is less than the A and $V a r_{x y}$ is less than $\mathrm{V}$, then the block is consider being lungs. Finally, the training patches are segmented. When the segmented area in the each segregated part is less than the certain ratio, the area is considered as the background. It is adaptive in nature.

Fig. 1 Schematic Diagram
The overall CNN consists of 50 convolution layers, 3 pooling nodes, 16 identity layer and one fully connected (FC) layer. The convolution operation is achieved with a 7 * 7 kernels and 35 training echoes and hyper parameters to certain range. The core aspect of a convolution operation is exchange of weight. The output of convolution layer passes through Rectified Linear Unit (ReLU) activation function which is nonlinear in nature. The output of convolution layer is given to the $4 * 4$ MaxPooling that executes standard down-sampling operation that decreases the plane dimension of feature maps such that minor shifts and distortions are introduced and the amount of corresponding learning parameters are decreased. The identity block contains two $1 \times 1$ convolution and one $3 \times 3$ convolution block and all the convolution output are added at last. This process is repeated for 16 times. The feature maps are translated into single dimension by average pooling. The final stage of the CNN is the fully connected layer. The obtained single-dimension average polling is connected to the FC layers. The FC layer subnet maps the features derived in the previous layers into the final network outputs including the probability of classification work for each level. Finally, 1-D probability is passed through activation function which is totally different from the other layers. Hence, the activation function used in this layer is softmax function. The function of softmax is to normalize the output real values. From this layer, the output is obtained whether the patient is COVID or not. At the time of testing, the grid method for extracting the feature is not required when utilizing the FCN [16, 17]. On comparison with the grid method, it doesnt required segmentation of image at the testing time. The final determination about the Covid and non-covid is done by comparing the probabilities values and the threshold value. The value of optimal threshold is determined by eliminating and normalizing method. The patches are available in the infected CT image which are used for differentiating the infected and disinfected patients. By comparing the test and training images,

\section{Fig. 1 Schematic Diagram}

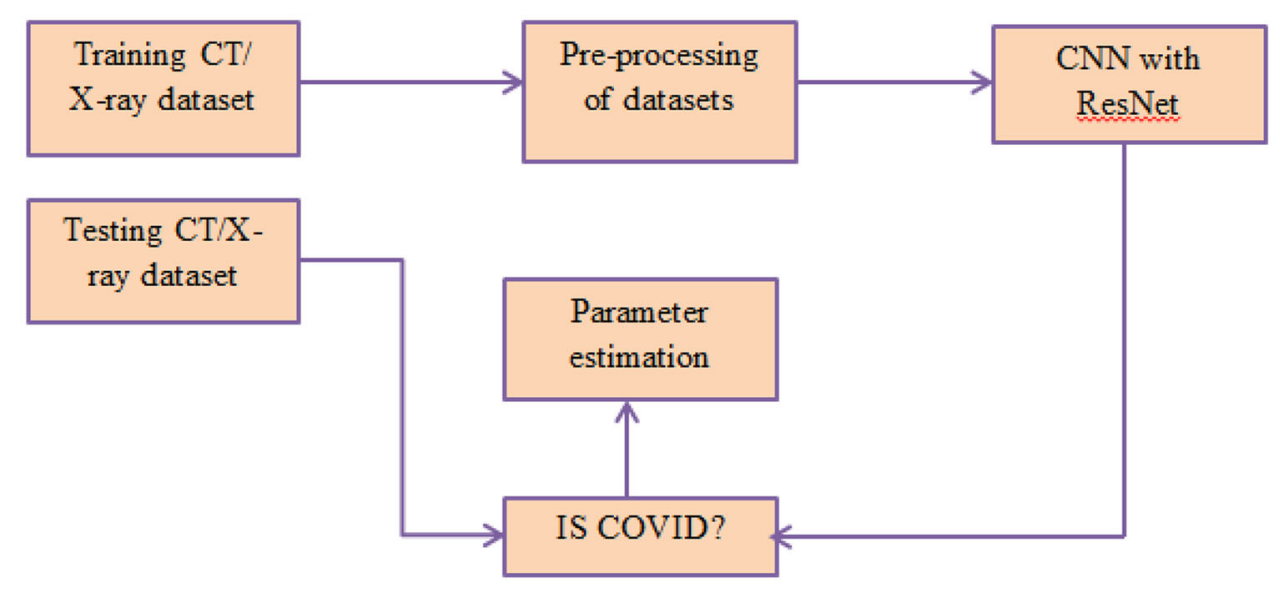




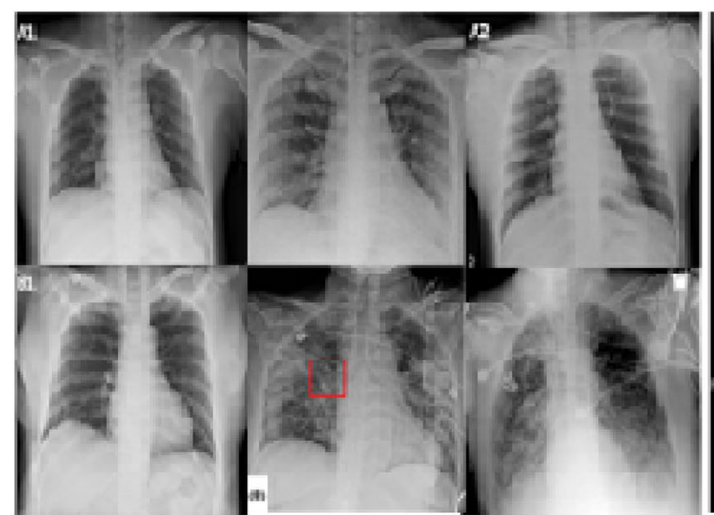

(a)

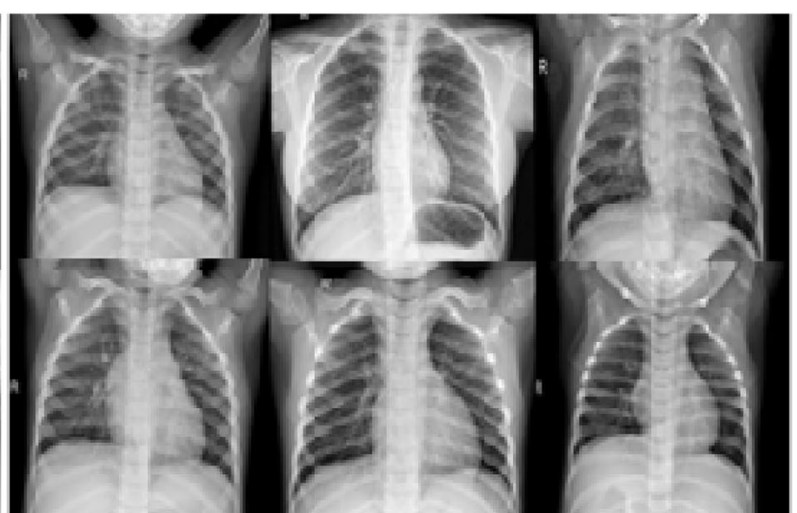

(b)

Fig. 2 a COVID-affected CT image; b Reference CT image

the COVID19-affected patient is diagnosed. The accuracy and specificity are obtained $95.09 \%$ and $81.56 \%$, respectively, on the selected sample dataset based on the CT images without the inclusion of another set of data such as geographical location, population density, etc. Also, the high sensitivity is also obtained as denoted in Table 1 . The CNN architecture is compared in terms of their accuracy. In proposed method, the ResNet architecture is used. The accuracy parameter of ResNet is compared with the other architectures such as Alexnet, ZFNet, GoogLe Net and VGG Net. The accuracy obtained for our data is tabulated in Table 1. Among all the architecture, ResNet seems to have higher accuracy(95.09\%). Eventhough VGG Net is the most commonly used architecture in CNN, it has less accuracy compared to the ResNet. Using the proposed method, COVID-19 positive patients are identified accurately and indicated as true positive patients whereas nonaffected patients are indicated as true negative. The parameters such as the accuracy, sensitivity, period for diagnosis and specificity are compared with both CT scan method and RT-PCR method in Table 1. It is inferred that the CT scan with ResNet CNN architecture provides more precision as compared with the RT-PCR.
Chest CT imaging can be accurate, realistic and speedy approach of diagnosing and testing COVID-19, particularly in comparison with RT-PCR. Hence, the image-based detection of COVID-19-affected patients is suggested. The proposed method gives the accuracy of $95.09 \%$ with a smaller number of images, and it is user friendly for the physician who can interpret medically. In comparison, around 60 percent of cases had standard CT characteristics associated with COVID-19, and nearly all patients had positive nucleic acid tests [18]. In the other side, "falsepositive" result in the nucleic acid test may give truepositive result in CT scan. $95.09 \%$ of the patients were initially positive for the chest CT which was compatible with COVID-19 before the initial positive RT-PCR report based on repeated RT-PCR tests and CT scans [18]. Chest Xray may also be considered for performing this task. It is concluded that the chest CT imagery has a strong susceptibility to COVID-19 treatment. To screen COVID-19, the CT scan should be considered, thorough assessment and follow-up, especially in crisis areas.

Table 1 Parameter comparison for RT-PCR method and CT scan method

\begin{tabular}{llllll}
\hline Method & Accuracy & & Period & Sensitivity & Specificity \\
\cline { 2 - 3 } RT-PCR & 95\% (After 4 tests) [8] & Requires 2-4 h for each test & Low & High \\
\hline & Architecture & Percentage & & \\
& Alexnet & 78.85 & & & High \\
& ZFNet & 79.54 & & High \\
\hline
\end{tabular}




\section{References}

1. Journal GH (2020) Interpretation of the diagnosis and treatment plan of Corona virus disease 2019 (tentative sixth edition)

2. Sardar R, Satish D, Birla S, Gupta D (2020) Comparative analyses of SAR-CoV2 genomes from different geographical locations and other coronavirus family genomes reveals unique features potentially consequential to host-virus interaction and pathogenesis. bioRxiv

3. Paraskevis D, Kostaki EG, Magiorkinis G, Panayiotakopoulos G, Sourvinos G, Tsiodras S (2020) Full-genome evolutionary analysis of the novel corona virus (2019-nCoV) rejects the hypothesis of emergence as a result of a recent recombination event. Infection Genetics Evol 79:104212

4. Journal GH (2020) Diagnosis and treatment plan of corona virus disease 2019 (tentative sixth edition) pp 1-5

5. Lu H, Stratton CW, Tang YW (2020) Outbreak of pneumonia of unknown etiology in Wuhan, China: the mystery and the miracle. J Med Virol 92(4):401-402

6. Cui C, Fan S, Lei H, Qu X, Zheng D (2019) Deep learning-based research on the influence of training data size for breast cancer pathology detection. J Eng 2019(23):8729-8732

7. Gerard SE, Patton TJ, Christensen GE, Bayouth JE, Reinhardt JM (2019) FissureNet: a deep learning approach for pulmonary fissure detection in CT images. IEEE Trans Med Imaging 38(1):156-166

8. Jiang Z, Chen Y, Zhang Y, Ge Y, Yin FF, Ren L (2019) Augmentation of CBCT reconstructed from under-sampled projections using deep learning. IEEE Trans Med Imaging 38(11):2705-2715

9. Qi Q et al (2019) Label-efficient breast cancer histopathological image classification. IEEE $J$ Biomed Heal Inform 23(5):2108-2116
10. Feng Y et al (2019) A deep learning approach for targeted contrast-enhanced ultrasound based prostate cancer detection. IEEE/ ACM Trans Comput Biol Bioinform 16(6):1794-1801

11. Liao F, Liang M, Li Z, Hu X, Song S (2019) Evaluate the malignancy of pulmonary nodules using the 3-D deep leaky noisy-OR network. IEEE Trans Neural Netw Learn Syst 30(11):3484-3495

12. Wang $Y$ et al (2020) Breast cancer image classification via multinetwork features and dual-network orthogonal low-rank Learning. IEEE Access 8:27779-27792

13. Masood A et al (2020) Cloud-based automated clinical decision support system for detection and diagnosis of lung cancer in chest CT. IEEE J Transl Eng Heal Med 8:1

14. Jiang $\mathrm{J}$ et al (2020) Multiple resolution residually connected feature streams for automatic lung tumor segmentation from CT images. IEEE Trans Med Imaging 38(1):134-144

15. Zhao J, Zhang, Y, He X, Xie P (2020) COVID-CT-Dataset: a CT scan dataset about COVID-19, arXiv Prepr. arXiv:2003.13865

16. Wang X, Hu Z (2017) Grid-based pavement crack analysis using deep learning. In: 2017 4th Int. conf. transp. inf. safety, ICTIS 2017-Proc., pp 917-924

17. Lu Q, Liu C, Jiang Z, Men A, Yang B (2017) G-CNN: object detection via grid convolutional neural network. IEEE Access 5:24023-24031

18. Ai T et al (2020) Correlation of chest CT and RT-PCR testing in coronavirus disease 2019 (COVID-19) in China: a report of 1014 cases. Radiology 2019:200642

Publisher's Note Springer Nature remains neutral with regard to jurisdictional claims in published maps and institutional affiliations. 